\title{
The influence of TSLP on the allergic response
}

\author{
MR Comeau ${ }^{1}$ and SF Ziegler ${ }^{2}$
}

Exposure to allergens first occurs at body surfaces in direct contact with the environment such as the skin, airways, and gastrointestinal tract, and compelling evidence suggests that allergic inflammatory responses are profoundly influenced by the products of epithelial cells located at these sites. One such product is thymic stromal lymphopoietin (TSLP), which is capable of affecting multiple cell lineages involved in allergic reactions. In this review we discuss recent work that has provided insight into the role TSLP plays in both aberrant and protective allergic inflammatory responses, as well as regulation, associations with disease, sources, and functions of this important cytokine.

\section{INTRODUCTION}

The increasing prevalence of diseases involving an allergic component is of global concern, accounting for a significant portion of annual healthcare expenditures worldwide. ${ }^{1,2}$ The most prevalent forms of allergic disease are allergic rhinitis, asthma, atopic dermatitis (AD), and food allergies. In general, allergy is characterized by an overreaction of the immune system to normally harmless foreign protein substances, known as allergens, following exposure through various routes such as inhalation, direct contact, injection, or ingestion. The symptoms of allergic reactions present themselves systemically and vary widely in their severity, with the organs and tissues involved dependent upon the point of contact with the allergen. ${ }^{3}$ Although some allergic conditions develop through IgE-independent pathways, ${ }^{2,4}$ allergy is primarily viewed as an IgE-mediated response that progresses from an immediate hypersensitivity reaction due to products of mast cell and basophil degranulation, to a latephase reaction characterized by leukocyte infiltration and swelling. ${ }^{5,6}$

The primary immune cell lineages involved in the initiation and progression of allergic inflammation include dendritic cells (DCs), mast cells, basophils, eosinophils, and type-2 helper T (Th2) cells. The responses of these principal players in allergic reactions are influenced by the local environments in which they reside. Immune cells localized within the epithelium at mucosal surfaces are at the site of primary exposure to pathogens and allergens. Consistent with this, recent evidence has demonstrated the importance of epithelial cross-talk with immune cells in developing innate and adaptive immune responses. In addition to providing a barrier to the external environment, epithelial cells express a variety of cell-surface and secreted factors in order to appropriately control immune responses. ${ }^{7,8}$ Among the various secreted factors epithelial cells are capable of producing, cytokines have emerged as having a broad influence on the development of allergic inflammation and many are targets of novel therapeutics currently in development. While several epithelial cell-derived cytokines are capable of influencing immune responses, one of the most thoroughly studied in the context of allergic inflammation is thymic stromal lymphopoietin (TSLP). The focus of this review will primarily be to discuss the role of TSLP in the cascade of events leading to allergic inflammation, the association of TSLP and gene variants with allergic disease, and to highlight recent progress that has been made in the identification of novel cell sources, functions, species-specific differences, and regulation of this potent cytokine.

\section{TSLP: AN EPITHELIAL CELL-DERIVED CYTOKINE ASSOCIATED WITH HUMAN ALLERGIC DISEASE}

TSLP is a member of the hematopoietic cytokine family that includes interleukin-2 (IL-2), IL-4, IL-7, IL-9, IL-13, IL-15, and IL-21. The initial studies to elucidate the biological activities of TSLP were focused on lymphopoiesis and similar to IL-7, TSLP was found to support mouse B-cell expansion both in vitro and in vivo. ${ }^{9-12}$ Using a variety of methods several groups identified a TSLP-binding protein in mouse, referred to as TSLP receptor (TSLPR), and showed that it was a low-affinity receptor for TSLP. Sequence analysis showed that TSLPR was similar to the common cytokine-receptor $\gamma$-chain $\left(\gamma_{c}\right) \cdot{ }^{13-15}$ Further analysis of

${ }^{1}$ Inflammation Research, Amgen Inc., Seattle, Washington, USA. ${ }^{2}$ Immunology Program, Benaroya Research Institute, Seattle, Washington, USA. Correspondence: MR Comeau (comeaumr@amgen.com).

Received 25 September 2009; accepted 16 November 2009; published online 16 December 2009. doi:10.1038/mi.2009.134 
the TSLP-receptor complex showed that the interleukin 7 receptor alpha (IL-7R $\alpha$ ) chain was a component of the high-affinity receptor, further linking these four-helix bundle cytokines. Later, in silico methods were used to isolate clones of human TSLP and TSLPR. ${ }^{16-18}$ The human proteins were found to be quite divergent from those in mouse at the sequence level, but, as described below, functionally they behave in a similar manner. Epithelial cells were found to be the principle source of TSLP, ${ }^{19}$ while several hematopoietic lineage cells were found to express both TSLPR and IL-7R $\alpha$, including DCs, monocytes, and T-cells. ${ }^{17}$

Studies of TSLP in humans demonstrated a potential role in Th2 inflammatory responses. The first supporting data along these lines were provided by a series of elegant experiments demonstrating several key findings: Primary human CD11 $\mathrm{c}^{+}$ myeloid DCs were found to coexpress the IL-7R $\alpha$ and TSLPR chains, and respond to TSLP stimulation by producing the CCR4binding, Th2 T-cell-attracting chemokines CCL17 and CCL22. Unlike other common DC-activating factors, TSLP-treated DCs are capable of priming naïve $\mathrm{CD} 4^{+}$helper T-cells to differentiate into proinflammatory Th2 T cells producing IL-4, IL-5, IL13 , and tumor necrosis factor- $\alpha$ (TNF- $\alpha$ ), but lower levels of interferon- $\gamma$ and IL-10. ${ }^{19}$ Additionally, TSLP was shown to be a potent $\mathrm{DC}$ survival and maturation factor inducing upregulation of surface HLA-DR, CD40, CD80, CD83, and CD86. ${ }^{17}$ Uniquely, TSLP induces OX40-L on DCs in the absence of IL-12, and the interaction between OX40 and OX40-L was identified as the molecular signal TSLP uses to prime naïve T-cells for Th2 differentiation. ${ }^{20,21}$ TSLP-treated DCs were also found to interact with $\mathrm{CRTH} 2{ }^{+} \mathrm{CD} 4{ }^{+} \mathrm{Th} 2$ memory T-cells to support their maintenance and further polarization, ${ }^{22}$ partially through upregulation of IL-17RB, conferring Th2-T-cell responsiveness to IL-25. ${ }^{23}$

It was established that in the steady-state TSLP is primarily expressed by epithelial cells in the skin, gut, and lungs; however, under inflammatory conditions, several addition cell types, including bronchial smooth-muscle cells and lung fibroblasts, express TSLP. ${ }^{19,24}$ In addition, TSLP protein was found to be highly expressed in the lesional skin of patients with $\mathrm{AD}$, but absent in normal skin or in skin samples from patients with Th1-type skin diseases, providing the first human disease association identified for TSLP. ${ }^{19}$ TSLP expression in acute and chronic lesions of AD skin is also associated with DC activation and migration. ${ }^{19}$ Together these seminal findings established TSLP as the first epithelium-derived cytokine with the capacity to skew the developing immune response toward a proallergic state through its direct actions on DCs.

\section{TSLP EXPRESSION INITIATES THE DEVELOPMENT OF ALLERGIC INFLAMMATORY DISEASE IN VIVO}

Human TSLP is found on chromosome 5q22.1, neighboring the atopy and asthma-associated cytokine cluster on $5 \mathrm{q} 31,{ }^{25}$ which encodes IL-3, IL-4, IL-5, IL-9, IL-13, and the IL-4 receptor. ${ }^{26}$ Like other Th2 cytokines its expression is associated with allergic inflammation in both mice and humans. The development of TSLP transgenic mice demonstrated that aberrant expression of this potent cytokine has dramatic local and systemic effects. Transgenic TSLP expression under the control of the lckproximal promoter, which is preferentially active during early lymphocyte development, ${ }^{27}$ results in systemic inflammatory disease involving the kidney, liver, spleen, lungs, and skin, and formation of cryoglobulins. Interestingly, these mice demonstrated progressive ulcerative lesions of the ears and a mixed perivascular leukocyte infiltrate in the lungs, ultimately leading to occlusion of the alveoli and death. ${ }^{28}$ Mice expressing TSLP under the control of the ubiquitous $\beta$-actin promoter also developed lethal systemic inflammation involving the bone marrow, spleen, thymus, and lungs. These mice display elevated serum IL-5 levels and myeloid hyperplasia as evidenced by the presence of myeloperoxidase-positive granulocytes in the spleen. As observed in lck-TSLP transgenic mice, the principal cause of death in $\beta$-actin TSLP transgenic mice was attributed to severely compromised lung function. ${ }^{29}$

Keratin-specific overexpression of TSLP in the skin under control of inducible $\mathrm{K}^{30}$ or constitutive $\mathrm{K} 14^{31}$ promoters lead to an AD-like phenotype, with the development of skin lesions containing inflammatory cell infiltrate, increased Th2 cyokines and chemokines in affected skin, and systemic increases in IgE. The results from inducible K5-TSLP transgenic animals are notable in their demonstration that mice born with normal TSLP levels may develop severe allergic inflammatory disease upon induction of TSLP. Similarly, in non-transgenic models, aberrant TSLP expression can be induced by keratinocyte-specific ablation of retinoid X receptors (RXR) or topical application of vitamin D3 and its low-calcemic analogues. This expression leads to the development of an $\mathrm{AD}$-like phenotype comparable to that seen in TSLP transgenic mice. ${ }^{31,32}$ Interestingly, the phenotype seen in both TSLP transgenic and vitamin D3-driven models was reported to be independent of T and B-cells, ${ }^{30,32}$ suggesting that the in vivo targets of TSLP at the sites of inflammation are likely myeloid-derived cells, which are then capable of initiating the disease process. ${ }^{30}$

As overexpression of TSLP in the skin leads to an AD-like phenotype, lung-specific overexpression of TSLP results in severe allergic airway inflammatory responses with asthmalike features. Mice overexpressing TSLP under the control of the lung-specific surfactant protein-C promoter (SPC-TSLP) develop Th2-biased CD4 ${ }^{+}$T-cell airway infiltrates, eosinophilia, increased serum IgE, airway hyper-responsiveness, and remodeling. ${ }^{33} \mathrm{~A}$ substantial reduction in airway inflammation and remodeling was observed in IL-4- or STAT6-deficient mice crossed with SPC-TSLP mice, or following blockade of IL-4 and IL-13 in SPC-TSLP mice with established airway disease. These results demonstrate that intact Th2 responses are necessary for the development of TSLP-induced airway inflammatory disease in this system. ${ }^{34}$

TSLP also plays a role in allergen-driven models of airway inflammation. In the ovalbumin-induced model of mouse asthma (OVA-asthma), TSLP expression is increased in response to antigen challenge and correlates with inflammatory cell infiltrates $^{33}$ and IL-5 levels in the broncho-alveolar lavage fluid. ${ }^{35}$ In this model TSLPR-deficient mice display greatly reduced airway inflammatory responses ${ }^{33,36}$ unless reconstituted with 
wild-type CD4 ${ }^{+}$T-cells. ${ }^{36}$ As well, blockade of TSLP using a TSLPR-Fc fusion protein ${ }^{36}$ or a TSLPR-blocking antibody ${ }^{35}$ significantly alleviated the allergic airway inflammatory response. Together these results highlight the involvement of TSLP signaling in this common model of asthma.

The profound phenotypes observed in mice overexpressing TSLP demonstrate the consequences of aberrant TSLP expression in vivo. Interestingly, administration of recombinant TSLP protein to normal mice reveals differences in systemic inflammatory responses and required mediators when compared with endogenous TSLP-induced phenotypes. Repeated intradermal TSLP administration over a 2-week time frame resulted in the development of a systemic Th2 response and a significant, diffuse inflammatory cell infiltrate in the skin containing eosinophils and mast cells, with subcutaneous fibrosis. ${ }^{37}$ In contrast to mice with dysregulated TSLP expression in the skin, ${ }^{30-32}$ in this model no skin lesions developed even after 6 weeks of TSLP administration (unpublished observations). Interestingly, while T-cells were found to be unnecessary for the phenotypes observed in mice expressing TSLP in the skin, ${ }^{30,32}$ they were a required component for the response induced following TSLP injection in the skin. ${ }^{37}$ In an acute model of lung inflammation, intranasal TSLP administration over a 2 -week period induced only a mild inflammatory response in the lung, unless co-administered with OVA as a model antigen ${ }^{38}$ upon which mice develop a robust airway inflammatory response. As well, OVA administration to SPC-TSLP transgenic mice prior to onset of spontaneous disease resulted in accelerated disease development. Taken together these results demonstrate the capacity of TSLP to drive the development of allergic inflammatory responses upon exposure to normally innocuous antigens. As observed in experiments involving injection of recombinant TSLP in the skin, ${ }^{37} \mathrm{~T}$-cells were found to be required for the TSLP + OVA-induced responses and contribute significantly to the phenotype that develops in the SPC-TSLP transgenic mice, indicating the requirement of an adaptive immune response for the complete TSLP-driven inflammatory response to develop in this model. ${ }^{38}$ Multiple factors could account for the differential responses seen in mice expressing native TSLP versus those receiving recombinant protein. Native mouse TSLP protein may have intrinsically different activities as compared with recombinant protein, as has been demonstrated with human TSLP. ${ }^{39}$ As well, the recombinant TSLP used in both exogenous administration studies contains 10 histidine residues at the $\mathrm{C}$-terminus of the protein, which may be seen as antigenic in mice repeatedly exposed to the protein. Alternatively, the constant systemic exposure to high levels of TSLP that occurs in transgenic and overexpression models may drive innate responses not seen with pulsatile delivery of recombinant protein. We have observed that intraperitoneally administered TSLP protein is no longer detectable in the circulation $2 \mathrm{~h}$ post injection (unpublished results). These caveats aside, both chronic and acute exposure to TSLP above homeostatic levels influences multiple aspects of the in vivo immune response, leading to the development of inappropriate allergic inflammatory responses.
Eczema in early life is often associated with the development of asthma and allergic rhinitis later in life, and this progression is referred to as the "atopic march". ${ }^{40}$ As demonstrated in transgenic mice, dysregulated TSLP expression can lead to the development of widespread and multi-focal inflammation, which may initiate in one organ and ultimately lead to disease in another tissue. The first data implicating TSLP with human asthma pathogenesis was provided by in situ hybridization studies. Increased numbers of TSLP-expressing cells were found in the epithelium and sub-mucosa of bronchial biopsies of patients with asthma as compared with normal controls, and this expression correlated with disease severity. ${ }^{41}$ An additional study of broncho-alveolar lavage fluid from moderate-to-severe asthmatics demonstrated elevated TSLP protein levels in asthma patients as compared with normal controls, ${ }^{42}$ suggesting that TSLP mRNA expression may translate into the presence of protein in patients with asthma.

Recently, a large international study of four heterogeneous asthma populations identified a genetic variant in the promoter region of the TSLP gene that was associated with protection from asthma, atopic asthma, and airway hyper-responsiveness. Associations between TSLP and asthma-related phenotypes were the most statistically significant observation in the study, which included over 5,500 genotyped individuals. ${ }^{43}$ An additional study identified a TSLP-gene variant associated with lower levels of allergen-specific IgE and total IgE in a gender-specific manner. ${ }^{44}$

Collectively, these studies provide compelling human disease association data and demonstrate that TSLP is necessary and sufficient for the initiation and development of allergic inflammation in rodents in vivo. The possibility that genetic variations affecting TSLP expression may influence asthmatic and allergic phenotypes across widespread populations, suggests an essential and central role for TSLP in the development of allergic inflammatory diseases.

\section{REGULATION OF TSLP EXPRESSION IN THE PERIPHERY}

Factors modulating TSLP expression encompass a wide variety of stimuli, which may be of cellular, microbial, or environmental origin, many of which are relevant to allergic inflammation. Constitutive TSLP expression is increased by the classic proinflammatory cytokines TNF- $\alpha$ and IL- $1 \alpha$ (or IL-1 $\beta$ ) in multiple cell types and tissues, ${ }^{19,39,45-49}$ and Th2 cytokines have also been shown to influence TSLP expression. When used alone, IL-4 and IL-13 are minimally effective at inducing TSLP expression, but when added in combination with either TNF- $\alpha$ or IL-1 demonstrate considerable synergy in human skin, lung, and gut samples. ${ }^{45,48,50}$ In contrast, IL-13 stimulation alone has recently been shown to induce robust TSLP production in mouse skin, lung, and nasal tissue cultures, suggesting that TSLP may be a downstream target of IL-13. ${ }^{51}$ Interestingly, IL-13 does not induce significant TSLP production in cultures of mouse small and large intestine. ${ }^{52}$ The promoters of both mouse and human TSLP contain nuclear factor- $\kappa \mathrm{B}$-binding sites that were found to be critical for TNF- $\alpha$ and IL- $1 \beta$-induced TSLP transcription, ${ }^{46}$ and mice with intestinal epithelial cell-specific deletion of IKK- $\beta$ show reduced TSLP expression, ${ }^{53}$ highlighting the importance of 
the nuclear factor- $\kappa B$ pathway in the regulation of TSLP expression. Additionally, members of the nuclear receptor superfamily are involved in the regulation of TSLP expression. In the skin, keratinocytes-specific ablation of the retinoid $\mathrm{X}$ nuclear receptor $\alpha$ and $\beta$ chains was shown to result in increased TSLP production in mice, leading to an $\mathrm{AD}$-like phenotype, a response also seen in mice topically treated with RXR agonists, vitamin D3, or its low-calcemic analogues. ${ }^{31,32}$

Microbial infections, pollution, and allergens are all known to exacerbate allergic responses. Consistent with this, epithelial TSLP expression has been shown to be increased following incubation with bacteria, ${ }^{24}$ infection of mice with the intestinal nematode Trichuris, ${ }^{54}$ stimulation with ligands for toll-like receptors (TLR) TLR2, TLR3, TLR8, and TLR9, ${ }^{39,45,46}$ rhinovirus infection, ${ }^{45}$ diesel exhaust particles, ${ }^{55}$ or cigarette smoke extract. ${ }^{56}$ While IgE-activated mast cells induce TSLP production from airway smooth-muscle cells in a TNF- $\alpha$-dependent manner, other products of mast cell activation, as well as common allergens and TLR ligands, fail to induce TSLP from these cells. ${ }^{49}$ Mediators of allergic responses are also known to regulate TSLP expression. Mast cells express TSLP mRNA, which is upregulated upon cross-linking of the IgE receptor, ${ }^{19}$ and pre-incubation with IL-4 results in significant upregulation of IgE-mediated TSLP protein and mRNA expression..$^{57}$ Recently, the contribution of proteases in regulating TSLP expression from both epithelial and hematopoetic cells has been reported. Proteases are a component of certain allergens and are also secreted by helminths. ${ }^{58}$ Proteases promote the development of Th2 reactions, ${ }^{59}$ and they are thought to provide a key link between Th2 immune responses in anti-helminth immunity and allergic responses. ${ }^{60} \mathrm{~A}$ study using the model protease allergen papain demonstrated its capacity to activate mouse basophils and induce TSLP mRNA and protein expression from these cells. ${ }^{61}$ Similarly papain and trypsin were shown to induce TSLP production from a human airway epithelial cell line. TSLP expression in this system was amplified in the presence of IL-4 and dependent upon the protease-activated receptor, PAR-2. ${ }^{60}$ Airborne allergens such as house dust mite are also associated with protease activity and administration of house dust mite to the airways of mice results in significant accumulation of TSLP in the broncho-alveolar lavage fluid. ${ }^{62}$

These recent advances in the understanding of factors and pathways that regulate TSLP have provided significant insight into scenarios that may lead to dysregulated TSLP expression in allergic disease, and demonstrate that numerous microorganisms and their products, known to be exacerbating factors for allergic disease, may induce TSLP in sufficient amounts to activate innate immune responses.

\section{TSLP AND EFFECTOR CELLS OFTHE ALLERGIC RESPONSE}

Initially the contributions of TSLP to the development of Th2biased immune responses were focused on its DC-specific activities. It is now clear that several other cell types involved in the allergic response are capable of responding to TSLP under permissive conditions (Figure 1).

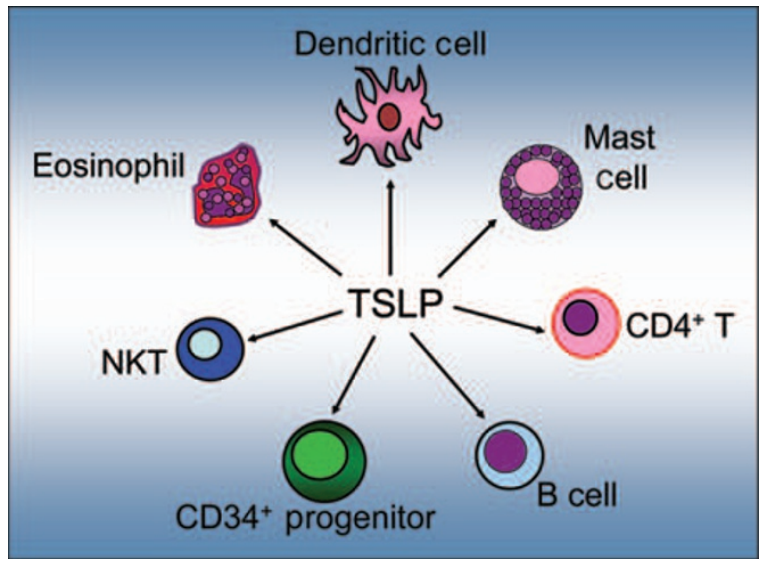

Figure 1 The constellation of TSLP-responsive cell types. TSLP derived from epithelial, stromal, and granulocytic cells acts on a variety of human and mouse cell populations, influencing multiple aspects of the allergic inflammatory response. TSLP, thymic stromal lymphopoietin.

\section{TSLP ANDTh2 T CELL RESPONSES}

Allergic inflammatory responses involve Th2 cell-derived cytokines and a common hallmark of affected tissues is the infiltration of $\mathrm{CD} 4{ }^{+} \mathrm{Th} 2$ cells. ${ }^{2}$ In addition to influencing Th2-T-cell differentiation indirectly through activation and programming of DCs, TSLP directly influences CD4 ${ }^{+}$T-cell differentiation into Th2 cells. TSLP was initially shown to induce proliferation of $\mathrm{CD} 4^{-} \mathrm{CD} 8^{-}$adult mouse thymocytes synergistically with IL- $1 \beta^{10}$ and TSLPR has been cloned from a Th2-skewed mouse T-cell library. ${ }^{15}$ Subsequently it was demonstrated that upon T-cell receptor (TCR) engagement mouse and human $\mathrm{CD} 4^{+} \mathrm{T}$-cells respond to TSLP stimulation, ${ }^{63-65}$ a response likely mediated through TCR-mediated upregulation of TSLPR expression. ${ }^{65}$ In experiments using cells sorted to greater than 99\% purity, TSLP was shown to induce the differentiation of mouse splenic $\mathrm{CD} 62 \mathrm{~L}^{\text {high }} \mathrm{CD} 4{ }^{+}$naïve T-cells into Th2 cytokineproducing cells that rapidly induced STAT5 phosphorylation and IL-4 production in response to TSLP. ${ }^{64}$ These data convincingly demonstrate that in the mouse, T-cells are direct targets of TSLP capable of responding in the absence of DCs. A recent study showed that TSLPR mRNA is not expressed in freshly isolated naïve, central memory, $\mathrm{CRTH} 2{ }^{+} \mathrm{CD} 4{ }^{+}$memory Th2, or effector memory human T-cell populations. ${ }^{66}$ In this study the authors did not determine whether TCR engagement conferred responsiveness to TSLP with these isolated T-cell populations as demonstrated in previous reports ${ }^{63-65}$ Although the contribution of contaminating DCs in T-cell experiments has recently been called into question, ${ }^{67,68}$ collectively these data suggest that T-cell activation is an additional mechanism by which TSLP is able to contribute to the developing immune response in the absence of DCs.

\section{BEYOND DCs; TSLP IS A POTENT ACTIVATOR OF HUMAN MAST CELLS}

Along with DCs, mast cells are located at sites exposed to the external environment such as the skin, airways, and gut, where they serve as crucial sentinel cells in host defense. ${ }^{69}$ Originally thought of only in terms of their contributions to immediate 
hypersensitivity reactions, the involvement of mast cells in innate and adaptive immune responses is now evident. ${ }^{70-74}$ Mast cell numbers in normal tissues vary depending on their anatomic location, and the severity of allergic responses are influenced by their concentration in tissues. ${ }^{3}$ Additionally, increased numbers of mast cells have been demonstrated in a variety of autoimmune and inflammatory conditions. ${ }^{75-77}$ Among human hematopoietic cells examined to date, mast cells are one of the only identified non-epithelial sources of TSLP. In addition to providing a potential source of TSLP in response to IgE-receptor cross-linking, ${ }^{19,57}$ mast cells express the functional TSLPR complex and respond to TSLP in the presence of IL- 1 and TNF- $\alpha^{39}$ or IL-33, ${ }^{78}$ suggesting that a proinflammatory environment is necessary to confer mast cell responsiveness to TSLP. Interestingly, native TSLP protein derived from both primary lung epithelial cells and lesional skin samples from AD patients has been shown to potently activate mast cells without inducing degranulation. TSLP stimulation induced the release of several cytokines (IL-5, IL-6, IL-13, TNF- $\alpha$, and granulocyte-macrophage colony-stimulating factor) and chemokines (CCL1 and CXCL8), but did not induce the release of pre-formed, granule-associated mediators such as $\beta$-hexosaminidase, histamine, leukotriene $\mathrm{C}_{4}$, and prostaglandin- $\mathrm{D}_{2} \cdot{ }^{39}$ These data implicate TSLP in IgE-independent forms of asthma and eczema, and suggest that in addition to DCs, mast cells at epithelial surfaces may be activated by TSLP, thereby contributing to both the initiation and perpetuation of innate immune responses.

Extending these findings, a potential role for TSLP as a mediator of cross-talk between bronchial smooth-muscle and mast cells was identified. In asthmatic patients, mast cells are the predominant inflammatory cells that accumulate within airway smooth-muscle-cell bundles. ${ }^{79}$ Mast cells play a key role in the orchestration of airway inflammation through their release of mediators capable of inducing bronchoconstriction, smoothmuscle-cell proliferation, and recruitment and activation of inflammatory cells. ${ }^{80,81}$ TSLP mRNA is expressed constitutively in cultured human bronchial airway smooth-muscle cells, ${ }^{19}$ while protein expression is increased upon stimulation with the proinflammatory cytokines TNF- $\alpha$ and IL- $1,{ }^{47}$ but not with Th2 cytokines IL-4 and IL-13. ${ }^{49}$ Additionally, individually or in combination, TNF- $\alpha$ and IL- 1 induce TSLP production from a variety of primary human cell types and tissues ${ }^{19,39,47-49}$ Unlike other cellular sources of TNF- $\alpha$ in allergic inflammation, mast cells are known to contain abundant pre-formed TNF- $\alpha$ stores, which are available for immediate release upon appropriate stimulation, 6,82 suggesting that in IgE-dependent reactions mast cells may represent a critical initial source of this proinflammatory cytokine. ${ }^{5}$ Supernatants from IgE-activated mast cells induce bronchial smooth-muscle cell production of TSLP in a TNF- $\alpha$ dependent manner and in turn bronchial smooth-muscle cell -derived TSLP is sufficient to induce mast cell production of IL-5 and IL-13. ${ }^{49}$ Consistent with these data, mast cells were shown to be necessary for TSLP expression in a model of allergic rhinitis using mast cell-deficient mice. ${ }^{83}$ Collectively these data suggest a potential feedback loop where in an allergic state, IgEactivated mast cells may provide the proinflammatory environment necessary for TSLP production possibly through release of

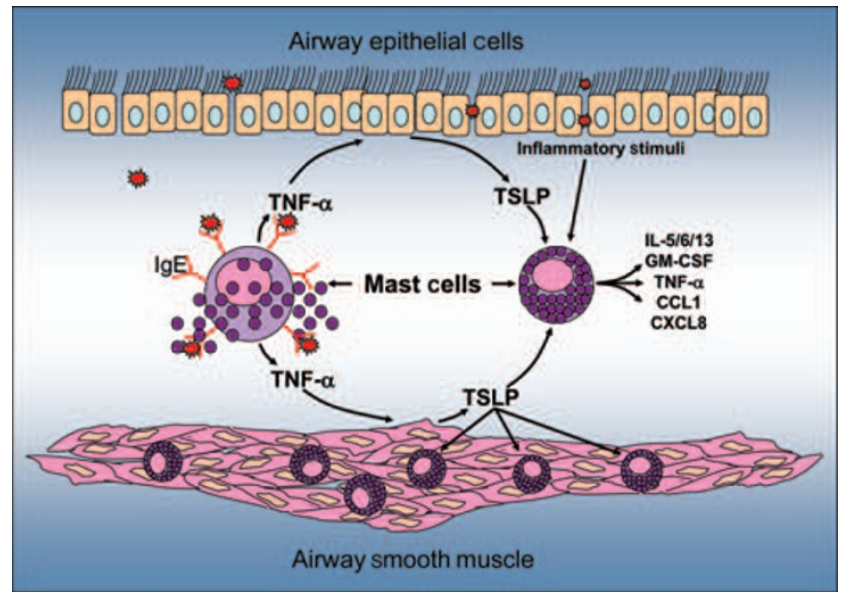

Figure 2 TSLP-mediated cross-talk between mast cells and airway epithelial and smooth-muscle cells in both IgE-dependent and independent inflammatory responses. Proinflammatory stimuli leading to epithelial cell production of TSLP directly activates mast cells, inducing release of multiple proinflammatory cytokines and chemokines independently of $\lg \mathrm{E}$, whereas IgE-mediated mast cell activation leads to release of TNF- $\alpha$, which may drive TSLP production from smooth-muscle cells in the airway. TNF- $\alpha$, tumor necrosis factor- $\alpha$; TSLP, thymic stromal lymphopoietin.

TNF- $\alpha$. In this environment TSLP may activate bystander mast cells contributing to the exacerbation of allergic inflammatory responses (Figure 2).

\section{THE EFFECTS OF TSLP ON PROGENITOR CELLS INTHE PERIPHERY}

The bone marrow is an active participant in systemic allergic inflammatory responses as several granulocytic effector cell types involved in allergic inflammation develop from CD34 ${ }^{+}$ bone marrow-derived progenitor cells. ${ }^{84}$ While differentiation and maturation of eosinophils and basophils from these progenitors primarily occurs within the bone marrow, mast cell maturation typically occurs in peripheral tissues. ${ }^{5} \mathrm{CD} 34^{+}$progenitor cells are normally present in circulation due to constant release from the bone marrow, ${ }^{85}$ and in allergic individuals increased numbers of progenitor cells are present in both the bone marrow and peripheral blood. Upon allergen exposure these progenitors traffic into local tissues where they are capable of maturing into mast cells, eosinophils, and basophils depending on their local environments. ${ }^{84,86,87}$ In mouse, TSLPR mRNA is expressed in hematopoietic progenitor cells and is upregulated in response to granulocytic differentiation signals. ${ }^{88}$ In humans, $\mathrm{CD} 34^{+}$progenitor cells express TSLPR mRNA ${ }^{78}$ and protein. ${ }^{89}$ As observed with mature mast cells, ${ }^{39}$ these progenitors respond to TSLP in the presence of co-stimulation with TNF- $\alpha$ and IL-1 or IL$33,{ }^{78}$ and rapidly produce abundant amounts of cytokines (IL-5, IL-13, granulocyte-macrophage colony-stimulating factor, IL-6) and chemokines (CXCL8, CCL1, CCL17, CCL22). Supernatants from nasal explant cultures of chronic rhinosinusitis patients have also been shown to induce the production of IL-5 from CD34 ${ }^{+}$progenitor cells in a TSLP-dependent manner. ${ }^{89} \mathrm{CD} 34^{+}$ progenitor cells residing in or recruited to tissues exposed to allergens may, therefore, contribute to proinflammatory 
processes following exposure to locally expressed, epitheliumderived cytokines such as TSLP and IL-33. ${ }^{89}$

\section{BASOPHILS AND EOSINOPHILS}

Along with mast cells, basophils and eosinophils are the primary effector cells of immediate hypersensitivity reactions and allergic disease. ${ }^{3}$ In addition, basophils and eosinophils are implicated in the pathogenesis of numerous inflammatory processes, including parasitic helminth infections. Present as mature cells found primarily in circulation, these cells can respond to a variety of activating stimuli and rapidly migrate to sites of inflammation. Both cell types are capable of a variety of immune functions and release an array of cytokines, chemokines, toxic granule proteins, and lipid mediators, thus participating as potent effector cells in the exacerbation of inflammatory responses. ${ }^{90-92}$ That TSLP is capable of activating mast cells and the common progenitors of eosinophils and basophils in the presence of proinflammatory signals, suggests there may be similar cofactors or scenarios where eosinophil and basophil responses to TSLP might occur. Indeed two recent reports have emerged describing direct activities of TSLP on human eosinophils. Peripheral blood eosinophils were found to express TSLPR and IL-7R $\alpha$ at the message and protein level, and respond to TSLP in a dose-dependent and specific manner. ${ }^{93}$ As well, eosinophil responses to TSLP were synergistically enhanced in the presence of IL-3 and TNF- $\alpha .{ }^{94}$ Interestingly, although TSLP induced the release of inflammatory cytokines (TNF- $\alpha$ IL-6, IL-8) and chemokines (CCL2, CCL3, CCL4, CXCL1, CXCL8), it did not induce degranulation of eosinophils, ${ }^{93,94}$ similar to what has been observed with mast cells. ${ }^{39}$ Additionally, both local and systemic allergic inflammatory responses to exogenous TSLP administration in the mouse were shown to involve eosinophils. Repeated intradermal administration of TSLP protein induces a systemic inflammatory response that is largely Th2 in nature ${ }^{37}$ and is characterized by systemic increases in circulating IgE, local inflammatory cell infiltrates, and increased Th2 cyokines and chemokines in the skin. Eosinophil-deficient dblGATA ${ }^{95}$ mice failed to develop both local and systemic responses to TSLP ${ }^{37}$ in this system. Collectively these recent data imply that in both humans and mice, eosinophils have the capacity to contribute to TSLP-driven allergic responses.

Although basophils constitute less than $1 \%$ of circulating leukocytes, these rare cells have potent effects on multiple aspects of the allergic inflammatory response. Recent findings have provided new insight into the role of basophils in allergic disease and immunity to helminths, suggesting that these cells may provide unique functions unmet by other hematopoietic cells. ${ }^{92,96}$ Although no direct responses to TSLP have yet been described, basophils have been identified as a potentially important source of TSLP in vivo. Mice exposed to the model protease allergen papain developed Th2 inflammatory responses with increased systemic IgE levels and transient appearance of IL-4 and TSLPproducing basophils in lymph nodes. Depletion of basophils in this model demonstrated their necessity in the differentiation of naïve CD4 T cells to Th2 cells. Additionally, basophils stimulated in vitro with papain expressed TSLP mRNA, while neutralization of TSLP in vivo inhibited papain-induced Th2 responses. ${ }^{61}$ As well delivery of recombinant TSLP protein leads to robust elicitation of basophils and their accumulation in the periphery unlike other epithelium-derived cytokines such as IL25 and IL-33. ${ }^{97}$ Taken together, these results suggest that in the context of protease allergens, basophil-derived TSLP is necessary for initiation of Th2 responses along with IL-4, and ascribe a potentially unique attribute to TSLP in comparison with other epithelium-derived cytokines that influence allergic inflammatory responses. Although no examination of basophils in mice overexpressing TSLP has been reported, these results suggest that they are likely a component of the lethal systemic phenotype observed in these animals. Thus, expansion of basophils under conditions that lead to dysregulated expression of TSLP may be a crucial early cellular response in the cascade of events leading to allergic inflammation.

\section{NKT CELLS}

A role for CD1d-restricted natural killer (NK)T cells has been proposed in asthma and although their contribution remains controversial, a significant body of supporting data provides a compelling case. ${ }^{98}$ Mouse invariant NKT (iNKT) cells were found to express both chains of the TSLPR and in the presence of TCR stimulation, proliferate in response to TSLP. ${ }^{99}$ While the proliferative response required TCR stimulation, similar to the TCR engagement required to confer TSLP responsiveness to CD4 $4^{+}$T-cells, ${ }^{63-65}$ TSLP alone was able to induce IL-13 production from iNKT cells. ${ }^{99}$ While iNKT cells preferentially produced IL-13 upon TCR engagement, IL- 4 and interferon- $\gamma$ were also abundantly produced in the presence of TSLP. When SPC-TSLP transgenic mice were crossed with mice lacking iNKT cells, no difference in the spontaneous development of allergic inflammatory responses was observed, suggesting that iNKT cells are not required for the phenotype observed in the TSLP transgenic mice. Interestingly, in a standard OVA-asthma model iNKT-deficient SPC-TSLP mice demonstrated significantly reduced airway hyperresponsiveness and IL-13 production, but no difference in pulmonary eosinophilia or IgE when compared with controls. ${ }^{99}$ It was therefore concluded that iNKT cells are required for SPC-TSLP mice to develop AHR in response to allergen challenge. Future studies will be required to determine whether human iNKT cells respond to TSLP in a similar manner.

\section{A ROLE FORTSLP IN GUT HOMEOSTASIS}

Although allergic reactions to normally innocuous antigens can result in damaging inflammation, Th2 immune responses are thought to have developed partially in order to protect the host from parasitic helminths, ${ }^{100}$ clearly a beneficial aspect of this arm of the immune response. TSLP is constitutively expressed by epithelial cells in the intestine, with the highest levels found in the proximal large intestine. ${ }^{24,53,54,101} \mathrm{~A}$ critical role for TSLP in the development of protective immunity to Trichuris muris infection was identified in mice with reduced TSLP expression due to an intestinal epithelial cell-specific deletion of IKK- $\beta .^{53}$ Reduced epithelial TSLP expression in these mice was associated 
with increases in pathogen-specific IL-12/23p 40, IL-17, and interferon- $\gamma$, and increased worm burdens as compared with those in control mice. Similar Th1 responses were observed in TSLPR-deficient mice, which also failed to expel worms. ${ }^{53}$ Extending these observations, it was reported that TSLP neutralization in normally genetically resistant mice also resulted in increased susceptibility to Trichuris infection and reduced Th2 cytokine production in the gut. Notably, blockade of interferon- $\gamma$ in TSLPR-deficient mice restored Th2 responses and immunity to Trichuris, demonstrating that TSLP is dispensible for the generation of protective Th2 cytokine responses in the intestine. ${ }^{101}$ Although the contribution of TSLP was less pronounced, similar results were obtained in two additional studies using helminth infection models. ${ }^{102,103}$ Consistent with a role for TSLP at limiting inflammation in the intestine, TSLPRdeficient mice develop more severe inflammation, increased weight loss, and elevated Th1 cytokines in the dextran sodium sulfate model of colitis. ${ }^{101}$ Interestingly, TSLPR-deficient mice also demonstrate reduced Th2 responses coupled with exaggerated IL-12 mRNA expression in the OVA-asthma model. ${ }^{36}$ Collectively these results suggest a principal function of TSLP in the intestine is to limit the production of non-protective Th1 cytokines and inflammation.

\section{SPECIES-SPECIFIC ACTIVITIES}

The IL-7R $\alpha$ chain is required for TSLP and IL-7 signaling as it is used by both cytokines in their receptor complexes. Mice deficient in the IL-7R $\alpha$ chain demonstrate impaired T and B-cell development, ${ }^{104}$ leading to deficiencies in both populations, and treatment of mice with neutralizing antibodies to IL-7 results in a similar phenotype. ${ }^{105}$ In contrast, mice lacking the TSLPR exhibit normal $\mathrm{T}$ and B-cell development and cellularity. ${ }^{63,106}$ IL-7 uses the common cytokine-receptor $\gamma$-chain $\left(\gamma_{c}\right)$ as an additional component of its receptor complex. ${ }^{107,108}$ Injection of TSLP into $\gamma_{c}$-deficient mice enhances the expansion of both $\mathrm{T}$ and $\mathrm{B}$-cells, and interestingly, mice lacking both the TSLPR and $\gamma_{c}$ display more severe lymphoid defects than $\gamma_{c}$-deficient mice. ${ }^{63}$ As IL-7 and TSLP both use the IL-7R $\alpha$ chain, these results suggest there may be a role for TSLP in mouse T and B-cell lymphopoiesis, although IL-7 likely plays the dominant role. Additionally, humans with severe combined immunodeficiency due to IL-7R $\alpha$ mutations lack T-cells but have normal B-cell numbers, ${ }^{109}$ a significant difference when compared with mice lacking the IL-7R $\alpha$ chain. While most of the identified TSLP activities have been demonstrated in both mouse and human systems, these findings suggest that like IL-7, speciesspecific activities exist for TSLP.

At the amino-acid level there are additional differences in TSLP to note between species. While the position of the six cysteine residues involved in disulfide bond formation are conserved across species, mouse and human TSLP share only $43 \%$ amino-acid identity overall. ${ }^{10,16}$ We have determined that this low level of conservation is also seen in additional rodent TSLP sequences, including rat (45\%) and rabbit (58\%) (Figure 3). Not surprisingly, non-human primate TSLP displays a much higher level of sequence conservation, with an amino-acid

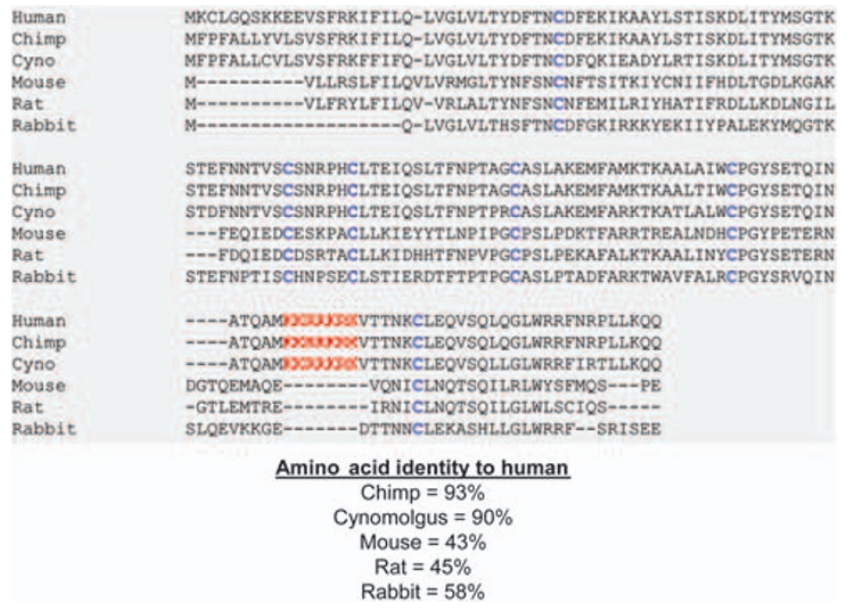

Figure 3 Species differences in the amino-acid sequences of TSLP. Primate TSLP contains a putative furin cleavage site upstream of the final conserved cysteine residue that is not present in rodent sequences. TSLP, thymic stromal lymphopoietin.

identity of $93 \%$ for chimpanzee and $90 \%$ for the cynomolgus monkey. A comparison of TSLP amino-acid sequences from several species reveals an interesting conservation in the primate sequences of seven basic amino acids (KKRRKRK) upstream of the final cysteine residue near the C-terminal end of the protein ${ }^{16}$ that is not present in any rodent sequence identified to date (Figure 3 ). This stretch of amino acids encodes a putative furin cleavage site. ${ }^{110}$ Furin is a proprotein convertase enzyme that is typically involved in the post-translational processing of inactive precursor proteins into their biologically active forms, an ancient mechanism that enables cells to regulate the levels of bioactive proteins. ${ }^{110}$ The presence of this conserved furin site in primate TSLP leads one to speculate on what purpose it may serve, if any, in the proteins biological activity. In an inflammatory state, furin cleavage may be a mechanism used by the primate immune system to limit the levels of bioactive TSLP protein in order to prevent inappropriate inflammatory responses. In order to determine whether this cleavage occurs and has any effect on TSLP activity, native TSLP expression would need to be examined both in the steady state and under inflammatory conditions where TSLP is thought to play a role.

\section{CONCLUDING REMARKS}

Much progress has been made in the understanding of the biological responses mediated by TSLP in the approximately 10 years since this cytokine was first cloned. Future studies of the interplay of TSLP with additional epithelium-derived cytokines such as IL-25 and IL-33 will likely reveal additional cellular targets and exciting novel findings. The regulation of TSLP by disease-relevant environmental factors, endogenous proinflammatory cytokines, and effector cells of the allergic response highlight the relevance of this epithelium-derived cytokine in innate and adaptive immunity (Figure 4) and make it an especially attractive target to consider for therapeutic intervention under both atopic and non-atopic conditions. 


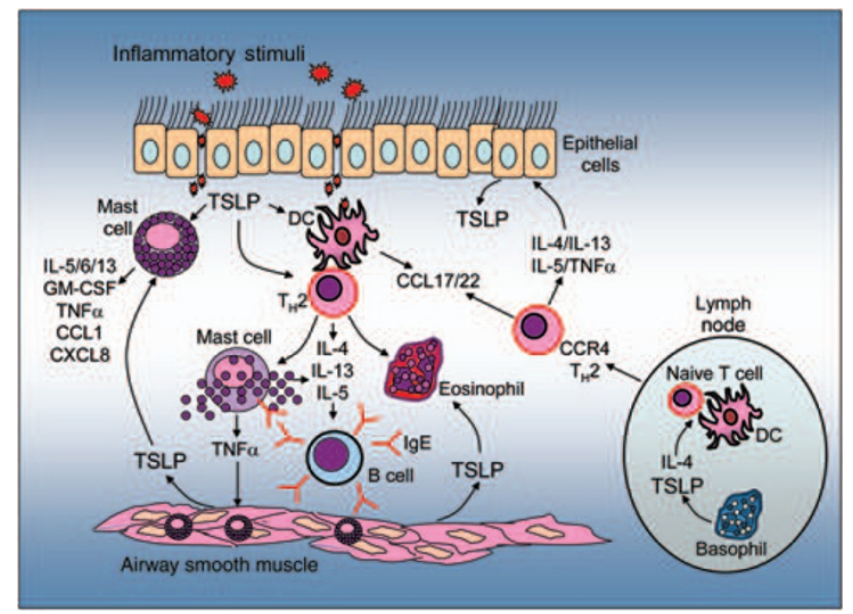

Figure 4 TSLP activation of key immune cells supports the development of allergic inflammatory responses. Multiple environmental and pathogenic factors are capable of inducing TSLP production from epithelial cells, which may act as both an upstream and downstream mediator of inflammatory responses through activation of inflammatory cells of the innate and adaptive immune response. Recently, activated basophils have been identified as an early source of IL- 4 and TSLP, which may serve to influence the differentiation of Th2 cells from naïve T-cells. IL, interleukin; Th2, type-2 helper T-cells; TSLP, thymic stromal lymphopoietin.

\section{ACKNOWLEDGMENTS}

We thank Heidi K. Jessup for excellent scientific contributions and critical review of the manuscript.

\section{DISCLOSURE}

Michael R. Comeau is an employee and shareholder of Amgen Inc.

C) 2010 Society for Mucosal Immunology

\section{REFERENCES}

1. Weiss, K.B. \& Sullivan, S.D. The health economics of asthma and rhinitis. I. Assessing the economic impact. J. Allergy Clin. Immunol. 107, 3-8 (2001).

2. Kay, A.B. Allergy and allergic diseases - first of two parts. N. Engl. J. Med. 344, 30-37 (2001).

3. Abbas, A. \& Lichtman, A. Cellular and Molecular Immunology. 5th edn (Saunders, Elsevier, 2005).

4. Humbert, M. et al. The immunopathology of extrinsic (atopic) and intrinsic (non-atopic) asthma: more similarities than differences. Immunol. Today 20, 528-533 (1999).

5. Costa, J.J., Weller, P.F. \& Galli, S.J. The cells of the allergic response: mast cells, basophils, and eosinophils. JAMA 278, 1815-1822 (1997).

6. Galli, S.J. \& Costa, J.J. Mast-cell-leukocyte cytokine cascades in allergic inflammation. Allergy 50, 851-862 (1995).

7. Saenz, S.A., Taylor, B.C. \& Artis, D. Welcome to the neighborhood: epithelial cell-derived cytokines license innate and adaptive immune responses at mucosal sites. Immunol. Rev. 226, 172-190 (2008).

8. Schleimer, R.P., Kato, A., Kern, R., Kuperman, D. \& Avila, P.C. Epithelium: at the interface of innate and adaptive immune responses. J. Allergy Clin. Immunol. 120, 1279-1284 (2007).

9. Friend, S.L. et al. A thymic stromal cell line supports in vitro development of surface lgM+ B cells and produces a novel growth factor affecting $B$ and T lineage cells. Exp. Hematol. 22, 321-328 (1994).

10. Sims, J.E. et al. Molecular cloning and biological characterization of a novel murine lymphoid growth factor. J. Exp. Med. 192, 671-680 (2000).

11. Levin, S.D. et al. Thymic stromal lymphopoietin: a cytokine that promotes the development of lgM+B cells in vitro and signals via a novel mechanism. J. Immunol. 162, 677-683 (1999).

12. Ray, R.J., Furlonger, C., Williams, D.E. \& Paige, C.J. Characterization of thymic stromal-derived lymphopoietin (TSLP) in murine B cell development in vitro. Eur. J. Immunol. 26, 10-16 (1996).
13. Park, L.S. et al. Cloning of the murine thymic stromal lymphopoietin (TSLP) receptor: Formation of a functional heteromeric complex requires interleukin 7 receptor. J. Exp. Med. 192, 659-670 (2000).

14. Isaksen, D.E. et al. Requirement for stat5 in thymic stromal lymphopoietinmediated signal transduction. J. Immunol. 163, 5971-5977 (1999).

15. Fujio, K. et al. Molecular cloning of a novel type 1 cytokine receptor similar to the common gamma chain. Blood 95, 2204-2210 (2000).

16. Quentmeier, H. et al. Cloning of human thymic stromal lymphopoietin (TSLP) and signaling mechanisms leading to proliferation. Leukemia 15, 1286-1292 (2001).

17. Reche, P.A. et al. Human thymic stromal lymphopoietin preferentially stimulates myeloid cells. J. Immunol. 167, 336-343 (2001).

18. Tonozuka, Y. et al. Molecular cloning of a human novel type I cytokine receptor related to delta1/TSLPR. Cytogenet. Cell Genet. 93, $23-25$ (2001).

19. Soumelis, V. et al. Human epithelial cells trigger dendritic cell mediated allergic inflammation by producing TSLP. Nat. Immunol. 3, 673-680 (2002).

20. Ito, T. et al. TSLP-activated dendritic cells induce an inflammatory $T$ helper type 2 cell response through OX40 ligand. J. Exp. Med. 202, 1213-1223 (2005).

21. Seshasayee, D. et al. In vivo blockade of OX40 ligand inhibits thymic stromal lymphopoietin driven atopic inflammation. J. Clin. Invest. 117, 3868-3878 (2007).

22. Wang, Y.H. et al. Maintenance and polarization of human TH2 central memory $T$ cells by thymic stromal lymphopoietin-activated dendritic cells. Immunity 24, 827-838 (2006).

23. Wang, Y.H. et al. IL-25 augments type 2 immune responses by enhancing the expansion and functions of TSLP-DC-activated Th2 memory cells. J. Exp. Med. 204, 1837-1847 (2007).

24. Rimoldi, M. et al. Intestinal immune homeostasis is regulated by the crosstalk between epithelial cells and dendritic cells. Nat. Immunol. 6 , 507-514 (2005).

25. Huston, D.P. \& Liu, Y.J. Thymic stromal lymphopoietin: a potential therapeutic target for allergy and asthma. Curr. Allergy Asthma Rep. 6, 372-376 (2006)

26. Walley, A.J., Wiltshire, S., Ellis, C.M. \& Cookson, W.O. Linkage and allelic association of chromosome 5 cytokine cluster genetic markers with atopy and asthma associated traits. Genomics 72, 15-20 (2001).

27. Wildin, R.S. et al. Developmental regulation of Ick gene expression in T lymphocytes. J. Exp. Med. 173, 383-393 (1991).

28. Taneda, S. et al. Cryoglobulinemic glomerulonephritis in thymic stromal lymphopoietin transgenic mice. Am. J. Pathol. 159, 2355-2369 (2001).

29. Osborn, M.J. et al. Overexpression of murine TSLP impairs lymphopoiesis and myelopoiesis. Blood 103, 843-851 (2004).

30. Yoo, J. et al. Spontaneous atopic dermatitis in mice expressing an inducible thymic stromal lymphopoietin transgene specifically in the skin. J. Exp. Med. 202, 541-549 (2005).

31. Li, M. et al. Retinoid X receptor ablation in adult mouse keratinocytes generates an atopic dermatitis triggered by thymic stromal lymphopoietin. Proc. Natl. Acad. Sci. USA 102, 14795-14800 (2005).

32. Li, M. et al. Topical vitamin D3 and low-calcemic analogs induce thymic stromal lymphopoietin in mouse keratinocytes and trigger an atopic dermatitis. Proc. Natl. Acad. Sci. USA 103, 11736-11741 (2006).

33. Zhou, B. et al. Thymic stromal lymphopoietin as a key initiator of allergic airway inflammation in mice. Nat. Immunol. 6, 1047-1053 (2005).

34. Zhou, B. et al. Reversal of thymic stromal lymphopoietin-induced airway inflammation through inhibition of Th2 responses. J. Immunol. 181, 6557-6562 (2008).

35. Shi, L. et al. Local blockade of TSLP receptor alleviated allergic disease by regulating airway dendritic cells. Clin. Immunol. (Orlando, FL) 129, 202-210 (2008).

36. Al-Shami, A., Spolski, R., Kelly, J., Keane-Myers, A. \& Leonard, W.J. A role for TSLP in the development of inflammation in an asthma model. J. Exp. Med. 202, 829-839 (2005).

37. Jessup, H.K. et al. Intradermal administration of thymic stromal lymphopoietin induces a T cell- and eosinophil-dependent systemic Th2 inflammatory response. J. Immunol. 181, 4311-4319 (2008).

38. Headley, M.B. et al. TSLP conditions the lung immune environment for the generation of pathogenic innate and antigen-specific adaptive immune responses. J. Immunol. 182, 1641-1647 (2009).

39. Allakhverdi, Z. et al. Thymic stromal lymphopoietin is released by human epithelial cells in response to microbes, trauma, or inflammation and potently activates mast cells. J. Exp. Med. 204, 253-258 (2007). 
40. Burgess, J.A. et al. Does eczema lead to asthma? J. Asthma 46, 429436 (2009).

41. Ying, S. et al. Thymic stromal lymphopoietin expression is increased in asthmatic airways and correlates with expression of Th2-attracting chemokines and disease severity. J. Immunol. 174, 8183-8190 (2005).

42. Ying, S. et al. Expression and cellular provenance of thymic stromal lymphopoietin and chemokines in patients with severe asthma and chronic obstructive pulmonary disease. J. Immunol. 181, 2790-2798 (2008).

43. He, J.Q. et al. A thymic stromal lymphopoietin gene variant is associated with asthma and airway hyperresponsiveness. J. Allergy Clin. Immunol. 124, 222-229 (2009).

44. Hunninghake, G.M. et al. Sex-stratified linkage analysis identifies a female-specific locus for lgE to cockroach in Costa Ricans. Am. J. Respir. Crit. Care Med. 177, 830-836 (2008).

45. Kato, A., Favoreto, S. Jr, Avila, P.C. \& Schleimer, R.P. TLR3- and Th2 cytokine-dependent production of thymic stromal lymphopoietin in human airway epithelial cells. J. Immunol. 179, 1080-1087 (2007).

46. Lee, H.C. \& Ziegler, S.F. Inducible expression of the proallergic cytokine thymic stromal lymphopoietin in airway epithelial cells is controlled by NFkappaB. Proc. Natl. Acad. Sci. USA 104, 914-919 (2007).

47. Zhang, K. et al. Constitutive and inducible thymic stromal lymphopoietin expression in human airway smooth muscle cells: role in chronic obstructive pulmonary disease. Am. J. Physiol. 293, L375-382 (2007).

48. Bogiatzi, S.I. et al. Cutting edge: proinflammatory and Th2 cytokines synergize to induce thymic stromal lymphopoietin production by human skin keratinocytes. J. Immunol. 178, 3373-3377 (2007).

49. Allakhverdi, Z., Comeau, M.R., Jessup, H.K. \& Delespesse, G. Thymic stromal lymphopoietin as a mediator of crosstalk between bronchial smooth muscles and mast cells. J. Allergy Clin. Immunol. 123, 958960. e952 (2009).

50. Tanaka, J. et al. Proinflammatory Th2 cytokines induce production of thymic stromal lymphopoietin in human colonic epithelial cells. Digest. Dis. Sci. (2009) (e-pub ahead of print).

51. Miyata, M. et al. Thymic stromal lymphopoietin is a critical mediator of IL13-driven allergic inflammation. Eur. J. Immunol. (2009).

52. Miyagaki, T., Sugaya, M., Fujita, H., Saeki, H. \& Tamaki, K. Increased serum thymic stromal lymphopoietin levels in patients with cutaneous $T$ cell lymphoma. Clin. Exp. Dermatol. 34, 539-540 (2009).

53. Zaph, C. et al. Epithelial-cell-intrinsic IKK-beta expression regulates intestinal immune homeostasis. Nature 446, 552-556 (2007).

54. Humphreys, N.E., Xu, D., Hepworth, M.R., Liew, F.Y. \& Grencis, R.K. IL33 , a potent inducer of adaptive immunity to intestinal nematodes. $J$. Immunol. 180, 2443-2449 (2008).

55. Bleck, B., Tse, D.B., Curotto de Lafaille, M.A., Zhang, F. \& Reibman, J. Diesel exhaust particle-exposed human bronchial epithelial cells induce dendritic cell maturation and polarization via thymic stromal lymphopoietin. J. Clin. Immunol. 28, 147-156 (2008).

56. Nakamura, Y. et al. Cigarette smoke extract induces thymic stromal lymphopoietin expression, leading to $\mathrm{T}(\mathrm{H}) 2$-type immune responses and airway inflammation. J. Allergy Clin. Immunol. 122, 1208-1214 (2008).

57. Okayama, Y. et al. FcepsilonRI-mediated thymic stromal lymphopoietin production by IL-4-primed human mast cells. Eur. Respir. J. 34, 425-435 (2009).

58. Perrigoue, J.G., Marshall, F.A. \& Artis, D. On the hunt for helminths: innate immune cells in the recognition and response to helminth parasites. Cell. Microbiol. 10, 1757-1764 (2008).

59. Comoy, E.E. et al. The house dust mite allergen, Dermatophagoides pteronyssinus, promotes type 2 responses by modulating the balance between IL-4 and IFN-gamma. J. Immunol. 160, 2456-2462 (1998).

60. Kouzaki, H., O'Grady, S.M., Lawrence, C.B. \& Kita, H. Proteases induce production of thymic stromal lymphopoietin by airway epithelial cells through protease-activated receptor-2. J. Immunol. 183, 1427-1434 (2009).

61. Sokol, C.L., Barton, G.M., Farr, A.G. \& Medzhitov, R. A mechanism for the initiation of allergen-induced $\mathrm{T}$ helper type 2 responses. Nat. Immunol. 9, 310-318 (2008).

62. Hammad, H. et al. House dust mite allergen induces asthma via Toll-like receptor 4 triggering of airway structural cells. Nat. Med. 15, 410-416 (2009).

63. Al-Shami, A. et al. A role for thymic stromal lymphopoietin in CD4(+) T cell development. J. Exp. Med. 200, 159-168 (2004).
64. Omori, M. \& Ziegler, S. Induction of IL-4 expression in CD4(+) T cells by thymic stromal lymphopoietin. J. Immunol. 178, 1396-1404 (2007).

65. Rochman, I., Watanabe, N., Arima, K., Liu, Y.J. \& Leonard, W.J. Cutting edge: direct action of thymic stromal lymphopoietin on activated human CD4+ T cells. J. Immunol. 178, 6720-6724 (2007).

66. Corrigan, C.J. et al. Early production of thymic stromal lymphopoietin precedes infiltration of dendritic cells expressing its receptor in allergeninduced late phase cutaneous responses in atopic subjects. Allergy 64 , 1014-1022 (2009).

67. Liu, Y.J. TSLP in epithelial cell and dendritic cell cross talk. Adv. Immunol. 101, 1-25 (2009).

68. Lu, N. et al. TSLP and IL-7 use two different mechanisms to regulate human CD4+ T cell homeostasis. J. Exp. Med., jem.20090153 206, 2111-2119 (2009).

69. Marshall, J.S. Mast-cell responses to pathogens. Nat. Rev. 4, 787-799 (2004).

70. Galli, S.J., Maurer, M. \& Lantz, C.S. Mast cells as sentinels of innate immunity. Curr. Opin. Immunol. 11, 53-59 (1999).

71. Galli, S.J. et al. Mast cells as "tunable" effector and immunoregulatory cells: recent advances. Ann. Rev. Immunol. 23, 749-786 (2005).

72. Galli, S.J., Nakae, S. \& Tsai, M. Mast cells in the development of adaptive immune responses. Nat. Immunol. 6, 135-142 (2005).

73. Beaven, M.A. Our perception of the mast cell from Paul Ehrlich to now. Eur. J. Immunol. 39, 11-25 (2009).

74. Brown, J.M., Wilson, T.M. \& Metcalfe, D.D. The mast cell and allergic diseases: role in pathogenesis and implications for therapy. Clin. Exp. Allergy 38, 4-18 (2008).

75. Theoharides, T.C. \& Cochrane, D.E. Critical role of mast cells in inflammatory diseases and the effect of acute stress. J. Neuroimmunol. 146, 1-12 (2004).

76. Benoist, C. \& Mathis, D. Mast cells in autoimmune disease. Nature 420, 875-878 (2002).

77. Woolley, D.E. The mast cell in inflammatory arthritis. N. Engl. J. Med. 348, 1709-1711 (2003).

78. Allakhverdi, Z., Smith, D.E., Comeau, M.R. \& Delespesse, G. Cutting edge: the ST2 ligand IL-33 potently activates and drives maturation of human mast cells. J. Immunol. 179, 2051-2054 (2007).

79. Brightling, C.E. et al. Mast-cell infiltration of airway smooth muscle in asthma. N. Engl. J. Med. 346, 1699-1705 (2002).

80. Page, S., Ammit, A.J., Black, J.L. \& Armour, C.L. Human mast cell and airway smooth muscle cell interactions: implications for asthma. Am. J. Physiol. 281, L1313-1323 (2001).

81. Rossi, G.L. \& Olivieri, D. Does the mast cell still have a key role in asthma? Chest 112, 523-529 (1997).

82. Gordon, J.R., Burd, P.R. \& Galli, S.J. Mast cells as a source of multifunctional cytokines. Immunol. Today 11, 458-464 (1990).

83. Miyata, M. et al. Mast cell regulation of epithelial TSLP expression plays an important role in the development of allergic rhinitis. Eur. J. Immunol. 38, 1487-1492 (2008).

84. Denburg, J.A. et al. Systemic aspects of allergic disease: bone marrow responses. J. Allergy Clin. Immunol. 106, S242-246 (2000).

85. Messner, H.A. Human hematopoietic progenitor in bone marrow and peripheral blood. Stem Cells (Dayton, Ohio) 16 (Suppl 1), 93-96 (1998).

86. Cyr, M.M. \& Denburg, J.A. Systemic aspects of allergic disease: the role of the bone marrow. Curr. Opin. Immunol. 13, 727-732 (2001).

87. Fanat, A.I., Thomson, J.V., Radford, K., Nair, P. \& Sehmi, R. Human airway smooth muscle promotes eosinophil differentiation. Clin. Exp. Allergy 39, 1009-1017 (2009).

88. Hiroyama, T. et al. Molecular cloning and characterization of CRLM-2, a novel type I cytokine receptor preferentially expressed in hematopoietic cells. Biochem. Biophys. Res. Commun. 272, 224-229 (2000).

89. Allakhverdi, Z. et al. CD34+ hemopoietic progenitor cells are potent effectors of allergic inflammation. J. Allergy Clin. Immunol. 123, 472-478 (2009).

90. Rothenberg, M.E. \& Hogan, S.P. The eosinophil. Annu. Rev. Immunol. $24,147-174$ (2006)

91. Shi, H.Z. Eosinophils function as antigen-presenting cells. J. Leuk. Biol. 76, 520-527 (2004)

92. Sullivan, B.M. \& Locksley, R.M. Basophils: a nonredundant contributor to host immunity. Immunity 30, 12-20 (2009).

93. Wong, C.K., Hu, S., Cheung, P.F. \& Lam, C.W. TSLP induces chemotactic and pro-survival effects in eosinophils: implications in allergic inflammation. Am. J. Respir. Cell Mol. Biol. (2009) (e-pub ahead of print). 
94. Hiraguchi, Y., Hosoki, K., Nagao, M., Tokuda, R. \& Fujisawa, T. Thymic stromal lymphopoietin directly activates eosinophils. J. Allergy Clin. Immunol. 123, S250-S250 (2009).

95. Yu, C. et al. Targeted deletion of a high-affinity GATA-binding site in the GATA-1 promoter leads to selective loss of the eosinophil lineage in vivo. J. Exp. Med. 195, 1387-1395 (2002).

96. Falcone, F.H., Haas, H. \& Gibbs, B.F. The human basophil: a new appreciation of its role in immune responses. Blood 96, 4028-4038 (2000).

97. Perrigoue, J.G. et al. MHC class II-dependent basophil-CD4+ T cell interactions promote $\mathrm{T}(\mathrm{H}) 2$ cytokine-dependent immunity. Nat. Immunol. 10, 697-705 (2009).

98. Matangkasombut, P., Pichavant, M., Dekruyff, R.H. \& Umetsu, D.T. Natural killer T cells and the regulation of asthma. Mucosal Immunol. 2, 383-392 (2009).

99. Nagata, Y., Kamijuku, H., Taniguchi, M., Ziegler, S. \& Seino, K. Differential role of thymic stromal lymphopoietin in the induction of airway hyperreactivity and Th2 immune response in antigen-induced asthma with respect to natural killer T cell function. Int. Arch. Allergy Immunol. 144, 305-314 (2007).

100. Anthony, R.M., Rutitzky, L.I., Urban, J.F., Jr, Stadecker, M.J. \& Gause, W.C. Protective immune mechanisms in helminth infection. Nat. Rev. 7, 975-987 (2007).

101. Taylor, B.C. et al. TSLP regulates intestinal immunity and inflammation in mouse models of helminth infection and colitis. J. Exp. Med. 206, 655-667 (2009).
102. Massacand, J.C. et al. Helminth products bypass the need for TSLP in Th2 immune responses by directly modulating dendritic cell function. Proc. Natl. Acad. Sci. USA 106, 13968-13973 (2009).

103. Ramalingam, T.R. et al. Regulation of helminth-induced Th2 responses by thymic stromal lymphopoietin. J. Immunol. 182, 6452-6459 (2009).

104. Peschon, J.J. et al. Early lymphocyte expansion is severely impaired in interleukin 7 receptor-deficient mice. J. Exp. Med. 180, 1955-1960 (1994).

105. Grabstein, K.H. et al. Inhibition of murine B and T lymphopoiesis in vivo by an anti-interleukin 7 monoclonal antibody. J. Exp. Med. 178, 257-264 (1993).

106. Carpino, N. et al. Absence of an essential role for thymic stromal lymphopoietin receptor in murine B-cell development. Mol. Cell. Biol. 24, 2584-2592 (2004).

107. Noguchi, M. et al. Interleukin-2 receptor gamma chain: a functional component of the interleukin-7 receptor. Science (New York, NY) 262, 1877-1880 (1993).

108. Kondo, M. et al. Functional participation of the IL-2 receptor gamma chain in IL-7 receptor complexes. Science (New York, NY) 263, 14531454 (1994).

109. Puel, A., Ziegler, S.F., Buckley, R.H. \& Leonard, W.J. Defective IL7R expression in $\mathrm{T}(-) \mathrm{B}(+) \mathrm{NK}(+)$ severe combined immunodeficiency. Nat. Genet. 20, 394-397 (1998).

110. Duckert, P., Brunak, S. \& Blom, N. Prediction of proprotein convertase cleavage sites. Protein Eng. Des. Sel. 17, 107-112 (2004). 